\title{
Prevalence of Iron Deficiency and Iron Deficiency Anemia in Infants and Children and Treatment with Microencapsulated Iron II Fumarate and Supplied Ascorbic Acid as "Sprinkles"
}

\author{
Abdullah Al-Mamari, 1,2*, Mohammad A. Al-Hegami3 , Sadeq Al-Hag1, Mahmoud Al-Buryhi2,4, \\ Safa Al-Amawi' ${ }^{1}$ Leena Ahmed ${ }^{1}$, Lila Al-Awadi', Sarah Al-Jamal', Wafa Mohammad1, \\ Yasmin Mohammad1 \\ ${ }^{1}$ Department of Biological Sciences, Faculty of Science, IBB University, Ibb, Yemen \\ ${ }^{2}$ Faculty of Medical Sciences, Al-Yemenia University, Sana'a, Yemen \\ ${ }^{3}$ Science Department (Biology), Faculty of Education, Sana'a University, Sana'a, Yemen \\ ${ }^{4}$ Department of Pharmaceutics, Faculty of Pharmacy, Sana'a University, Sana'a, Yemen \\ Email: ${ }^{*}$ almamarynew@yahoo.co.in
}

Received 4 April 2014; revised 22 May 2014; accepted 12 June 2014

Copyright (C) 2014 by authors and Scientific Research Publishing Inc.

This work is licensed under the Creative Commons Attribution International License (CC BY).

http://creativecommons.org/licenses/by/4.0/

(c) (i) Open Access

\section{Abstract}

Background: Iron deficiency anemia (IDA) in children and infants is a common nutritional problem all over the world. Infants and young children have a high risk for developing iron deficiency (ID) because they have high demand for iron during the period of rapid growth. This is aggravated by the insufficiency of iron in their diet. Iron supplementation programs using pediatric tablets or drops have not been successful in the control of anemia amongst infants and children in some countries. "Sprinkles" is an innovative multi-micronutrient home fortification strategy to control iron deficiency and anemia to be more useful. Objective: The objective was to estimate the prevalence ID and IDA in infants and children in Ibb City, Yemen Republic; evaluate the use of a new form of iron and determine the hematologic response to different doses and forms of iron in Sprinkles and iron drops. Design: Using a prospective, randomized, controlled design, we studied 337 randomly children aged 24 - 48 months and infants aged 6 - 24 months in Ibb City (hemoglobin: $70-99 \mathrm{~g} / \mathrm{L})$. One group received a daily sachet of microencapsulated ferrous fumarate $(80 \mathrm{mg}$ elemental $\mathrm{Fe}$ ) in powder form plus ascorbic acid to be sprinkled onto any complementary food eaten (sprinkles group); a control group received ferrous sulfate drops 3 times/d for 2 months

\footnotetext{
${ }^{*}$ Corresponding author.
}

How to cite this paper: Al-Mamari, A., et al. (2014) Prevalence of Iron Deficiency and Iron Deficiency Anemia in Infants and Children and Treatment with Microencapsulated Iron II Fumarate and Supplied Ascorbic Acid as "Sprinkles". Pharmacology \& Pharmacy, 5, 716-724. http://dx.doi.org/10.4236/pp.2014.57081 
(total dose: 40 mg elemental Fe). Hemoglobin and serum ferritin concentrations were measured at baseline and at the end of treatment. Results: Successful treatment of anemia (hemoglobin $>100$ $\mathrm{g} / \mathrm{L}$ ) occurred in $58 \%$ of the sprinkles group and in $56 \%$ of the drops group, with minimal side effects in both groups. Geometric mean ferritin concentrations increased significantly in each group from baseline to the end of treatment $(P<0.001)$. Conclusion: Use of ferrous sulfate drops or a single daily dose of microencapsulated ferrous fumarate sprinkles plus ascorbicacid resulted in a similar rate of successful treatment of anemia without side effects. To our knowledge, this is the first demonstration of the use of microencapsulated iron sprinkles to treat anemia in this area. Improved ease of use may favor the use of sprinkles to deliver iron.

\section{Keywords}

Infants, Children, Anemia, Microencapsulated Iron, Ascorbic Acid

\section{Introduction}

Iron deficiency anemia is a leading cause of morbidity and mortality worldwide and affects up to two-thirds of children in most developing countries [1]. Infants and young children aged 6 - 18 months are particularly vulnerable to iron deficiency anemia because their requirement for iron is high [2]. "Sprinkles" is a novel form of microencapsulated iron, which can be packaged in ease to use sachets for fortifying infants' weaning foods at home [3] [4]. For a community setting, UNICEF recommends a daily dose of $12.5 \mathrm{mg}$ of iron (as ferrous sulfate drops) for children 6 - 24 months old [5]. However, the absolute amount of absorbed iron varies with the dose of iron, the form of iron compounds and different modes of delivery (drops, tablets, iron-fortified foods) for many reasons; adherence to treatment is poor despite multiple efforts to influence and improve it [6] [7]. At that meeting, a new method to provide micronutrients (including iron) to populations at risk was suggested. The intervention was based on 2 observations from the West, where micronutrient deficiencies are rare [8]. The observations are that the commercial food fortification works well to prevent deficiencies, and the fortificants must not appreciably change the color, texture, or taste of the food to which they are added [9]. Pharmaceutical encapsulation has been used for years to mask the strong taste of certain drugs and even vitamins [10]. The encapsulation is a thin coating of a soya-based hydrogenated lipid. Encapsulation prevents the micronutrients from oxidizing the food, which means that there is no change in the color or taste of the food [11]. To administer the correct amount of iron, the encapsulated micronutrients were packaged in single-dose sachets, as is the case with oral rehydration sachets [12]. To use the sachets, one is instructed to sprinkle the entire contents onto whatever food is being served in the household. In this prospective, randomized, controlled trial, we tested the hypothesis that the response to treatment of anemia would be better with 2 months of treatment with microencapsulated ferrous fumarate sachets daily than that with ferrous sulfate drops provided 3 times/d [13]. The objective in this study aimed to evaluate the use of a new form of iron and determine the hematologic response to different doses and forms of iron in Sprinkles and iron drops.

\section{Subjects and Methods}

\subsection{Study Area and Subjects}

The study took place between February 2012 to June 2013 in the field study area at Al-Thaurah general hospital and Motherhood and Childhood hospital in Ibb City. The prevalence of anemia in young children is estimated to be 70\%, a significant proportion of which is due to iron deficiency [14] [15] The CBC test it was performed also to provide information about the following measurements: (MCV), (MCH), (MCHC). Because these values reflect the size and hemoglobin concentration of individual cells and is useful in diagnosing different types of anemia [22]. While the second tube used to determine serum iron level by Spectrophotomaterically using the commercially available kits it is iron liquid color and measure serum ferritin concentration. All tests were analyzed at the Laboratory of Mother childhood Hospital, in Ibb City. The criteria for ID were $\mathrm{Hb}<11 \mathrm{~g} / \mathrm{dL}$, and $<10 \mathrm{~g} / \mathrm{dL}$ for IDA while the criteria of ferritin $<12 \mathrm{~g} / \mathrm{L}$ and MCV $<74 \mathrm{fL}$ for IDA and for ID ferritin $<12 \mathrm{~g} / \mathrm{L}$ and MCV $<85 \mathrm{fL}$. The serum iron criteria for IDA $<40 \mu \mathrm{g} / \mathrm{dl}$ and $<60-40 \mu \mathrm{g} / \mathrm{dl}$ for ID. In addition to periph- 
eral smear was taken for indicate the etiology of the anemia based on red cell morphology if microcytic hypochromic cells. Infant who had ferritin, MCV and $\mathrm{Hb}$ above these cutoff points were classified as iron-sufficient and low were classified as IDA. These values deemed to be appropriate for this age group. These values were used according to the WHO criteria [16]. To be included in the study, infants had to be $6-24$ months of age at the time of recruitment, be ingesting a weaning food in addition to breast milk, and have a hemoglobin concentration between 70 and $99 \mathrm{~g} / \mathrm{L}$, measured during a baseline assessment. Children who were severely anemic (hemoglobin $<70 \mathrm{~g} / \mathrm{L}$ ) were excluded from the trial and treated. Oral consent to conduct the study in the Ibb City was obtained from the mothersof the infants included in the study and hospitals data.

\subsection{Study Design and Protocol}

After the baseline assessment, children were randomly assigned to 1 of 2 treatment groups. The drops group received ferrous sulfate drops ( $5 \mathrm{mg}$ elemental $\mathrm{Fe} \cdot \mathrm{kg} 1 \cdot \mathrm{d} 1$, rounded to a total of $40 \mathrm{mg}$ elemental $\mathrm{Fe}$ ) provided in 3 equal doses/d. The Sprinkles group received microencapsulated ferrous fumarate (80 mg elemental Fe) packaged in a sachet with ascorbic acid (50 mg), which was added to the child's meal (after it was cooked) once daily. The Sprinkles were mixed and packaged. A single bottle of ferrous sulfate was dispensed to mothers in the drops group once every 4 weeks. Mothers in the Sprinkles group were provided with an adequate number of sachets for 2 weeks. The dosage of iron in the Sprinkles sachet was double that in the ferrous sulfate drops. On the basis of algorithms, which account for major enhancers and inhibitors of iron absorption, we estimated that the absorption of encapsulated iron (in the sachets) would be significantly lower than that of the nonencapsulated iron [10]-[16]. However, to our knowledge, no previous studies in humans have examined the absorption of hydrogenated soy lipid-coated ferrous fumarate Sprinkles. A recent study [17] reported that the mean absorption in infants of iron from standard ferrous fumarate at fortification levels in infant cereal was $4.1 \%$ (range: $1.7 \%$ $14.7 \%$ ). These data cannot be directly extrapolated to iron absorption from encapsulated ferrous fumarate for many reasons. In the current study, infants were anemic, suggesting higher absorption potential. However, we used references data in our algorithms, which estimated absorption of microencapsulated iron added to cereal to be in the range of $2 \%-8 \%$. Thus, the total amount of available iron in an $80 \mathrm{mg}$ sachet is $1.6-6.4 \mathrm{mg}$ [1]-[5]. There is little recent data on iron absorption in infants receiving ferrous sulfate drops. On the basis of a compilation of older data, we assumed that iron absorption would be in the range of $4 \%-18 \%$, making the total amount of available iron 1.6 - $7.2 \mathrm{mg} / \mathrm{d}$ [3]-[18]. Thus, the range of potentially absorbed iron was similar, but not identical, for the 2 forms of iron. Because the ferrous fumarate was coated, we anticipated that there would be minimal intestinal irritation from the relatively high $80-\mathrm{mg}$ dose of iron. During the baseline assessment, a written questionnaire was administered to collect demographic, nutritional, and health data for each infant. Field workers visited infants at 2-wk intervals after the baseline visit, for a total of 5 visits. At each visit, a questionnaire about side effects, ease of use, and compliance over the preceding $7 \mathrm{~d}$ was completed. Side effects included the incidence of diarrhea, constipation, and general discomfort after ingestion of the iron drops. Questions about ease of use included whether the children objected to taking the iron and whether microencapsulated ferrous fumarate changed the color, taste, or texture of the infants' food. To evaluate compliance during each visit, the number of used (empty) sachets was counted or unused drops measured. At each visit, fieldworkers provided parents with oral educational reinforcement to maximize compliance with the intervention. Anthropometric measurements were completed during the baseline and final visits. Capillary blood samples taken at the baseline and final visits were obtained from a finger prick with the use of aseptic techniques; hemoglobin concentrations were measured immediately with a portable hemcue B-hemoglobin photometer (Hemocue Inc., Angelholm, Sweden) by trained technicians using standardized techniques [19]. Malaria parasite smears were taken (at the baseline visit only) and $500 \mu \mathrm{L}$ blood samples were collected and preserved in ice-lined cold boxes. Blood samples were returned to the Laboratory within $6 \mathrm{~h}$ of collection, where the serum was separated by centrifugation $(12,000 \mathrm{~g}, 10 \mathrm{~min}$, room temperature) before storage at $-40^{\circ} \mathrm{C}$. Serum ferritin was assayed in duplicate with a commercial enzyme-linked immune-sorbentas-say (Spectro Ferritin Kit; Ramco Laboratories, Houston) [13]. Baseline and endof-treatment ferritin samples from an individual subject were assayed on the same day (in a single batch) on one 96-well microliter plate to minimize interassay variation. An external reference standard (Lyphochek Anemia Control; Bio-Rad, Anaheim, CA) was assayed in duplicate on each microliter plate for the ferritin assay [11].

\subsection{Sample Size and Power}

The primary outcome was the successful treatment of anemia the proportion of children with hemoglobin con- 
centrations $\geq 100 \mathrm{~g} / \mathrm{L}$. On the assumption of $90 \%$ cure rates in the drops group and $80 \%$ in the Sprinkles group and with a type I error set at 0.05 and a 0.9 probability of detecting a true difference, the final sample size estimate was 337 subjects per group. Whole blood samples collected at baseline and on day some samples were used to make thin blood smears. The remaining blood was frozen at $40^{\circ} \mathrm{C}$ for analysis of iron deficiency. It was our expectation that $65 \%$ of the infants in the Ibb region would have hemoglobin values within our target range (70 $99 \mathrm{~g} / \mathrm{L}$ ); therefore, a total of 131 infants were screened to obtain the needed number of subjects for the study.

\subsection{Data Processing and Analysis}

Data forms were manually checked for completeness and consistency before submission for processing. The proportion of children who were successfully treated was compared between the groups with chi-square analysis. Paired t tests were used to analyze the change in hemoglobin, ferritin and anthropometric measurements over time. Differences between groups in hemoglobin, ferritin and anthropometric measurements from the beginning to the end of the study were assessed with Student's t test and the interaction between treatment and time of the study was assessed with use of a two-factor, repeated-measures analysis of variance. The acceptable level of statistical significance for all tests was $\mathrm{P}<0.05$. Results are expressed as geometric means $( \pm$ SDs) and ranges.

\section{Results}

In Table 1, The results showed statistically very significant difference $(\mathrm{P}<0.05)$ between IDA prevalence and Children age we founded in infants aged between 5 weeks to 2 years numeral 20 (13\%) was ID and 91 (69\%) was IDA. While normal cases were $20(13 \%)$ respectively, totally 131 patient out of 337 patients studded. While prevalence of iron deficiency and iron deficiency anemia in children's aged between above 25 months to 48 months it was 14 (7\%) ID and 77 (37\%) IDA and number of normal cases were 115 (56\%) respectively, totally 206 patient out of 337 patients studded. Total of iron deficiency (ID) in this study were 34 case with (10\%) out of 337 case and iron deficiency anemia prevalence reached 168 (50\%) in our study. Overall, the results in this study showed that the infants more infected by IDA than children's. The value in prevalence of iron deficiency anemia it was taken according to third parameter criteria was used Hb level, MCV, ferritin or serum iron including Hct and blood film smear if microcytichypocromic (Figure 1) or macrocytichypochromic (Figure 2).

After the screening survey infants with hemoglobin concentrations between 70.0 and $99.9 \mathrm{~g} / \mathrm{L}$ and other criteria were randomly assigned to treatment consequently, a total 337 patients, 131 of them infants and 206 were children's completed the final assessment, including anthropometric measurements and blood sampling. In Table 2 results showed the effects on iron status as following there was no difference in baseline $\mathrm{Hb}$ across groups (range 8.60 to $8.90 \mathrm{~g} / \mathrm{dL}$ ). There was a significant rise in $\mathrm{Hb}$ at end of treatment 8 weeks (range 5.0 to 9.0 $\mathrm{g} / \mathrm{dL}$ ) and at 16 weeks (range 12.9 to $16.6 \mathrm{~g} / \mathrm{dL}$ ) in all groups with no significant differences across groups. Fifty-seven percent $57.7 \%$ of infants advanced from an anemic to a nonanemic state (hemoglobin $\geq 100 \mathrm{~g} / \mathrm{L}$ ). This rate was not significantly different between groups: $57.7 \%$ subjects in the Sprinkles group and $56.3 \%$ subjects in the drops group. The relative risk of remaining anemic after 4 months of treatment was 1.5 times greater in the drops group than in the Sprinkles group, but the difference was not significant. Data were also analyzed to determine the percentage of infants who positively responded to iron treatment; a positive response was defined as an increase in hemoglobin of $\geq 10 \mathrm{~g} / \mathrm{L}$ in the final blood sample). In the Sprinkles group, (67.5\%) of the children responded; but in the drops group, (56.7\%) responded. Overall, in both groups studded in our study, there was a significant increase in hemoglobin concentrations from baseline to the end of treatment study $(\mathrm{P}<0.001)$. Table 2 also shows the change in ferritin levels at start supplementation were similar across groups (range of median

Table 1. Percentage of prevalence of ID \& IDA anemia between infants and children's in all clinical samples studied in Ibb City.

\begin{tabular}{|c|c|c|c|c|c|c|c|c|c|}
\hline \multirow{2}{*}{\multicolumn{2}{|c|}{$\begin{array}{l}\text { According to age group } \\
\text { (months) }\end{array}$}} & \multicolumn{3}{|c|}{$\begin{array}{l}\text { Prevalence of anemic (ID \& IDA) and } \\
\text { non-anemic cases between Infants and } \\
\text { Children's group. Number (\%) }\end{array}$} & \multirow{2}{*}{$\begin{array}{l}\text { Total of } \\
\text { samples } \\
\text { examined }\end{array}$} & \multicolumn{3}{|c|}{ Chi-Square Test } & \multirow[t]{2}{*}{$\begin{array}{l}\text { Effect } \\
\text { of size }\end{array}$} \\
\hline & & ID & IDA & Normal & & Value & Degree of freedom & Significance & \\
\hline Infants & 5 weeks to 24 months & $20(13 \%)$ & $91(69 \%)$ & $20(13 \%)$ & 131 & $4.408^{\mathrm{a}}$ & 2 & 0.110 & 0.03 \\
\hline \multirow[t]{2}{*}{ Children } & 25 to 48 months & $14(7 \%)$ & 77 (37\%) & 115 (56\%) & 206 & & & & \\
\hline & Total & $34(10 \%)$ & $168(100 \%)$ & 135 (40\%) & 337 & & & & \\
\hline
\end{tabular}




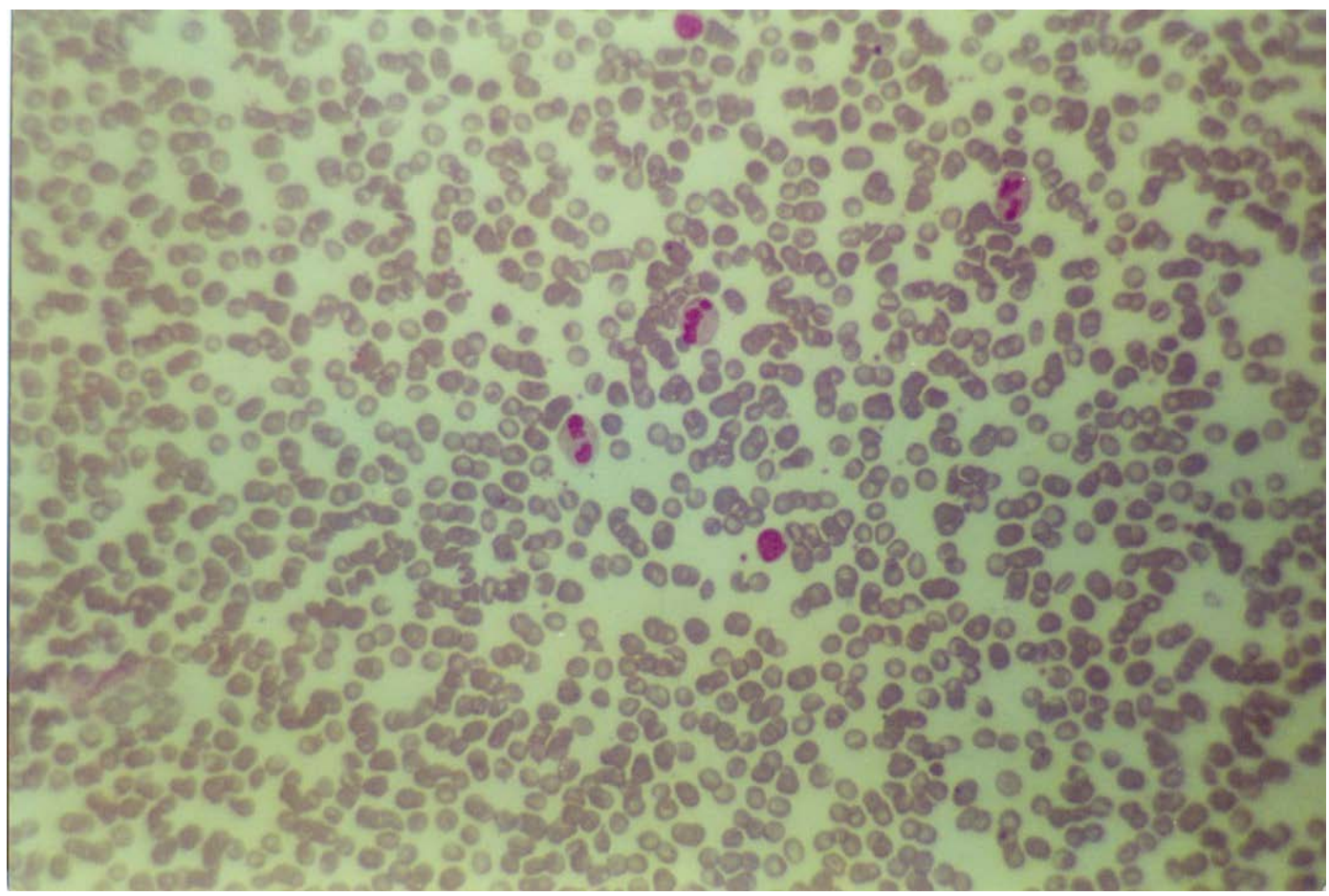

Figure 1. Blood smear of patient with severe iron deficiency anemia in infants.

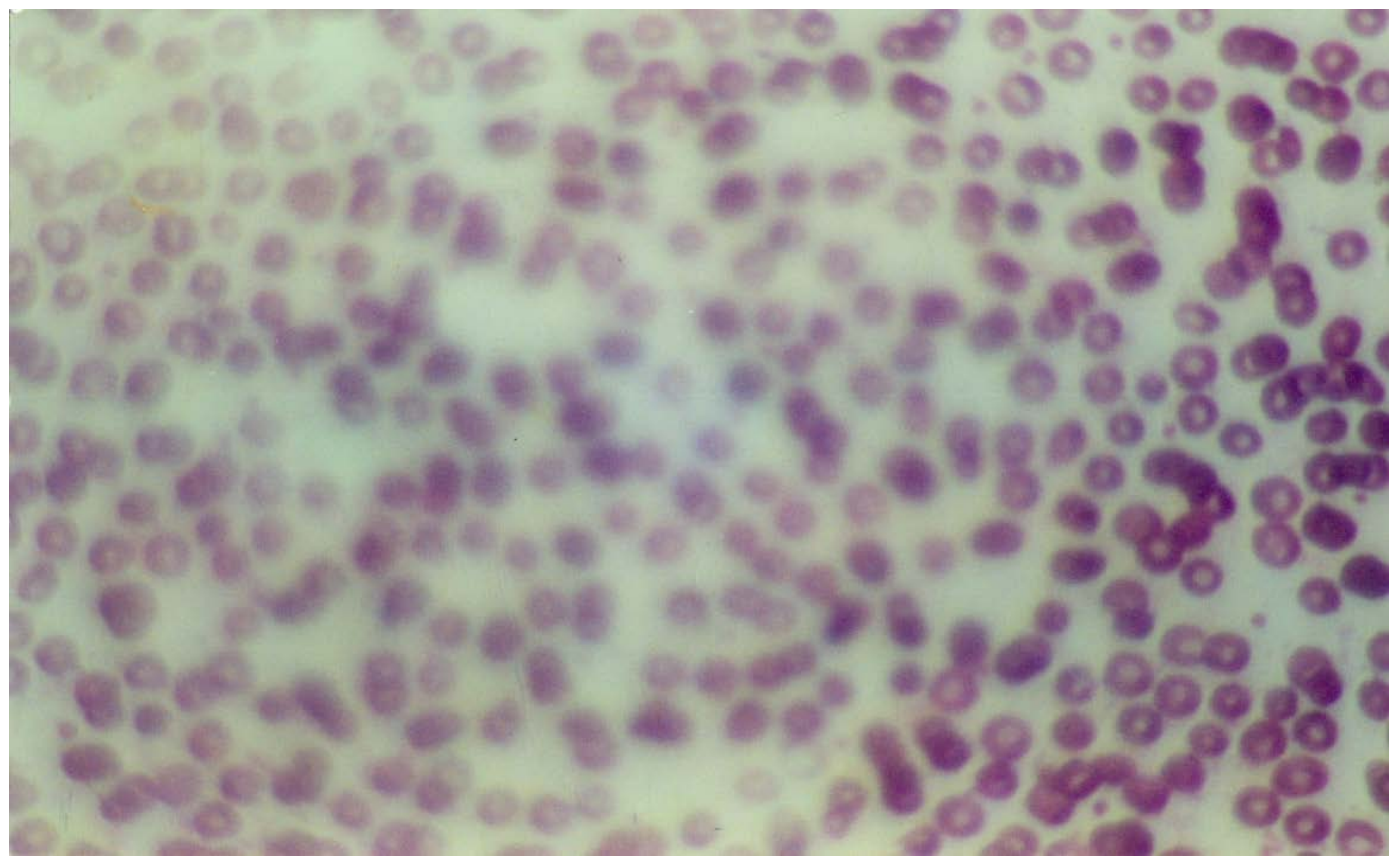

Figure 2. Blood smear of patient with moderate iron deficiency anemia in children.

from 2.5 to $4.6 \mathrm{ng} / \mathrm{mL}$ ). While, ferritin levels showed a significant increase in end of treatment after 8 weeks from baseline within each group with differences across groups. However, the mean value was significantly higher in the Sprinkles group than in the intervention drops group 10.1:7.5 respectively. The range of ferritin concentrations was wide at both baseline and at the end of supplementation, as is usual with the wide inter individual and analytic variance associated with this measure, especially in a region in which malaria and parasites are endemic [20]. 
The characteristics and baseline hematologic indexes of the infants who completed the study are shown below in Table 3 by iron status subgroup. Age, sex distribution, and anthropometric z scores (weight-for-age, heightfor-age, and weight-for-height) did not differ significantly between the infants in the 3 iron-status subgroups. Despite evidence suggesting the presence of iron deficiency in many of the subjects, most plasma ferritin concentrations were within the range expected for healthy subjects. No treatment effect was found for weight-forage or weight-for-height $\mathrm{z}$ scores in either group. There was a significant decrease in height-for-age $\mathrm{z}$ scores in both groups from baseline to the final measurements (change: $0.16 \pm 0.65 ; \mathrm{P}<0.001$ ).

Table 2. Hemoglobin and ferritin concentrations and the percentage of anemic cases, by treatment group, at baseline and after 2 months of treatment.

\begin{tabular}{|c|c|c|c|c|c|}
\hline \multirow{2}{*}{ Hb \& Fr. concentration with anemia \% } & \multirow{2}{*}{ Drops group } & \multicolumn{3}{|c|}{ Sprinkles group } & \multirow{2}{*}{ P-value } \\
\hline & & FF $12.5 \mathrm{mg}$ & FF $20 \mathrm{mg}$ & FF $12.5 \mathrm{mg}$ & \\
\hline \multicolumn{6}{|l|}{ Hb concentration (g/L) } \\
\hline Baseline & $8.64(0.79)$ & $8.90(0.71)$ & $8.84(0.79)$ & $8.71(0.82)$ & 0.20 \\
\hline $8 \mathrm{wk}$ & $9.59(1.41)$ & $9.41(1.28)$ & $9.31(1.61)$ & $9.6(1.1)$ & $0.62^{*}$ \\
\hline P-value (Base-3 wk) & $<0.0001$ & 0.0009 & 0.02 & $<0.0001$ & \\
\hline $16 \mathrm{wk}$ & $10.1(0.15)$ & $10.40(1.41)$ & $10.10(1.88)$ & $10.38(1.34)$ & $0.32^{*}$ \\
\hline P-value (Base-8 wk) & $<0.0001$ & $<0.0001$ & $<0.0001$ & $<0.0001$ & \\
\hline \multicolumn{6}{|l|}{ Percent Anemic (\%) } \\
\hline Baseline & 100 & 100 & 100 & 100 & \\
\hline $8 w k$ & 63.5 & 70.0 & 70 & 65.5 & $0.75^{*}$ \\
\hline $16 \mathrm{wk}$ & 46.6 & 36.1 & 45.9 & 38.2 & $0.78^{*}$ \\
\hline P-value (Base 8 wk) & $<0.0001$ & $<0.0001$ & $<0.0001$ & $<0.0001$ & \\
\hline \multicolumn{6}{|l|}{ Ferritin $(\mu \mathrm{g} / \mathrm{L})$} \\
\hline Baseline & $2.5(1-35.1)$ & $4.3(1-40.6)$ & $2.7(1-0.5)$ & $4.6(1-35.1)$ & $0.24^{* *}$ \\
\hline 8 weeks & $10.5(1-97.2)$ & $7.35(1-87.2)$ & $9.1(1-17.3)$ & $7.2(1-100.2)$ & $0.09^{\dagger+}$ \\
\hline$P$ value change $e^{\S}$ & $<0.0001$ & 0.19 & $<0.0001$ & 0.12 & \\
\hline
\end{tabular}

${ }^{++}$Hemoglobin g/dL: mean (SD); ${ }^{*}$ Adjusted for baseline $\mathrm{Hb} ;{ }^{\dagger} \mathrm{Hb}<10 \mathrm{~g} / \mathrm{dL} ;{ }^{\ddagger}$ Serum ferritin: median, (range); ${ }^{\S} \mathrm{P}$ value for log transformed ferritin change from baseline to $8 \mathrm{wk} ;{ }^{* *} \mathrm{P}$ value for log transformed ferritin; ${ }^{++} \mathrm{P}$ value for log transformed ferritin adjusted for baseline log ferritin.

Table 3. Infants characteristics and baseline iron and hematologic indexes.

\begin{tabular}{|c|c|c|c|c|}
\hline Infant characteristics & Iron deficiency anemia $(\mathrm{n}=91)$ & Iron deficiency $(\mathrm{n}=20)$ & Iron sufficiency $(\mathrm{n}=20)$ & P-value \\
\hline Age (mo) & 11.8 (6.2 - 18.6)a,2 & $14.4(8.9-18.6) b$ & $11.0(6.6-18.6) \mathrm{a}$ & 0.03 \\
\hline Sex $(\% M)$ & 53 & 23 & 55 & 0.2 \\
\hline Height-for-age (z score) & _0.5_ 1.0 (_2.7 to 1.4$) \mathrm{a}, 3$ & $\_0.7 \_1.0\left(\_3.2\right.$ to 1.5$) \mathrm{a}$ & _ $0.9 \_1.4$ (_4.6 to1.2)a & 0.4 \\
\hline Weight-for-age (z score) & _ $1.2 \_1.4($ (4.3 to 1.8$) \mathrm{a}$ & $\_1.6 \_0.9\left(\_4.0\right.$ to $\left.\_0.4\right) \mathrm{a}$ & _ $1.8 \_1.7$ (_6.2 to 0.8$) \mathrm{a}$ & 0.3 \\
\hline \multicolumn{5}{|l|}{ Baseline indexes } \\
\hline Hemoglobin $(\mathrm{g} / \mathrm{L})$ & $88.4 \_7.9(72-98) a$ & 107.7 _ $7.3(100-127) \mathrm{b}$ & $109.7_{-} 7.2(100-121) b$ & $<0.0001$ \\
\hline Anemia [n (\%)] & 91 (100)a & $0(0) \mathrm{b}$ & $0(0) \mathrm{b}$ & $<0.0001$ \\
\hline Ferritin $(\mu \mathrm{g} / \mathrm{L})$ & $17.9(4-166) \mathrm{a}$ & $38.7(11-287) b$ & $27.2(7-157) b$ & 0.01 \\
\hline Irondeficiency [n (\%)] & 20 (100)a & 20 (100)a & $0(0) \mathrm{b}$ & $<0.0001$ \\
\hline
\end{tabular}




\section{Discussion}

This is the first population-based descriptive epidemiological study to estimate the prevalence of ID and IDA in Ibb City infants and children's. We found that the prevalence of ID and IDA in infants aged 5 weeks - 24 months were 20 (13\%) 91 (69\%) respectively, which is disturbingly high. While the prevalence of ID and IDA in children's aged above 24 months - 4 years were 14 (7\%), 77 (37\%) respectively (Table 1). In the present study, totally the prevalence of IDA168 (49.8\%) (Table 1) was very concordant in comparison to the previous studies results and IDA is a common problem for both the developed and the developing world. In general in this side from our study, it can be concluded that infants and children's with low maternal educational level, poverty, health care, high proportions of calories in the diet from cow's milk and low nonheme iron density were the determinant factors for risk of anemia. Although the prevalence of IDA in this area of Yemen Republic is similar to that in most of the developed world, it still constitutes a public health problem. In the ideal dose of iron supplementation for treatment of IDA in community programs has long been an issue of debate. This double-blind cluster randomized clinical trial was conducted in a community setting to evaluate the hematological responses to various doses and forms of iron using Sprinkles versus traditional iron drops [21]. We used iron drops (ferrous glycine sulphate) in a dose equivalent to $20 \mathrm{mg}$ of elemental iron to match the dose recommended by the National Program for Control and Prevention of Anemia [16]. Since Sprinkles is to be mixed with food, the absorption of iron in Sprinkles may be affected by the ascorbic acid content of the weaning foods. On the other hand, it is known that in the presence of iron deficiency, higher amounts of iron from Sprinkles are absorbed even with food [12]-[17]. Hence, to determine the lowest efficacious dose of iron in Sprinkles, we evaluated 3 doses of iron (ferrous fumarate) in Sprinkles viz., $30 \mathrm{mg}, 20 \mathrm{mg}$ and $12.5 \mathrm{mg}$. Our study found a significant increase in hemoglobin levels as early as 8 and 16 weeks in all the groups with no differences across groups. It was at baseline 8.64 (0.79) while was at 8 weeks 9.59 (1.41) 9.41 (1.28), 9.31 (1.61), 9.6 (1.1) and 10.1 (0.15), 10.40 (1.41), 10.10 (1.88), 10.01 (1.33), 10.38 (1.34) at 16 weeks respectively (Table 2). Similarly, ferritin levels increased significantly in all groups at start of supplementation the mean was $3.4(1$ - 43.2) while was at end of treatment 10.5 (1 - 97.2), 7.35 (1 - 87.2), 9.1 (1 - 17.3), 9.1 (1 - 115.6), 7.2 (1 - 100.2) with no differences across groups (Table 2). The number of children with anemia also decreased significantly in all intervention groups from $100 \%$ at baseline while was at end of treatment as following 46.6, 36.1, 45.9, and 38.2. Therefore, we conclude that Sprinkles containing $12.5 \mathrm{mg}$ of ferrous fumarate are as efficacious as higher doses of Sprinkles or iron drops in raising hemoglobin levels 10.40 (1.41) in anemic children. In addition, parasite infections, diarrhea, gastroenteritis, respiratory infections and malaria were all significantly higher in the iron drops group when compared with Sprinkles group. Consequently, children with moderately severe anemia would likely have remained anemic if treatment were not provided. At the end of the 2 mo intervention, 65\% of infants remained anemic. There are many possible explanations for this observation parasites have been shown to be a significant contributor to the etiology of anemia, especially in young infants in highly endemic areas [22]. Parasitic and malaria, which are common in West Africa, may also contribute to continuing blood losses and bone marrow unresponsiveness [23]. Other endemic infections, such as gastroenteritis and respiratory infections might also interfere with the utilization of absorbed iron. Another possible reason for the lack of response to iron was a subclinical vitamin A deficiency because infants in Ibb City are known to be at risk of this deficiency. Previous epidemiologic studies indicated that vitamin-A deficiency and anemia often coexist [12]. It is likely that vitamin-A plays a role in mobilizing iron from stores to be used for hematopoiesis [11]. Thus, it is possible that those infants who did not respond to the drops or Sprinkles did not have iron deficiency anemia but had other causes of anemia. At the end of the 2 mo of treatment with iron, serum ferritin concentrations (an indirect measure of iron stores) had increased significantly in both groups. These results suggest that more iron was absorbed than was needed for immediate erythropoiesis. However, the changes in ferritin concentrations from baseline to the end of the study were greater in the drops group than in the Sprinkles group (Table 2). Despite the presence of ascorbic acid and the higher total dose of iron in the Sprinkles group, the bioavailability of iron was likely lower from the Sprinkles than from the drops. This observation is not surprising because the Sprinkles were mixed with food, which would likely impede iron absorption more than would drops that were taken without food. Increasing the dose per sachet of Sprinkles would likely offset this difference; however, this was not necessary for the treatment of anemia. One might argue that iron absorption would be enhanced if the iron dose in the Sprinkles were divided among 2 - 3 servings/d because fractional iron absorption is inversely related to the dose [24]. Our findings suggest important policy and program implications for the treatment of anemia. Although we did not measure compliance with the different dosing regimens directly (the protocol was not de- 
signed as an effectiveness study), our data suggest that compliance was sufficient to treat anemia and increase iron stores. Compliance with the Sprinkles (67\% of the group never missed a dose) was less than it was with the drops. There are many likely explanations for this difference. Many infants in the study who had just reached 6 mo of age at baseline were just starting to consume complementary foods (in addition to breastfeeding). Some of the infants at this age were not yet consuming these foods daily. Because Sprinkles had to be added to complementary foods, if these foods were not eaten the Sprinkles would not be provided. Finally, A further advantage of the Sprinkles is that more than one micronutrient can be included in the sachet, depending on the needs of a specific locale. For example, iron, ascorbic acid, vitamin A, zinc, and iodine can easily be combined and included in the sachet. The characteristics and baseline hematologic indexes of the infants who completed the study are shown in (Table 3) by iron status subgroup, age, sex distribution, and anthropometric (weight-for-age, height-for-age, and weight-for-height) there were no significant differences in age (14.9 $\pm 4.5 \mathrm{mo})$ or in hemoglobin or ferritin concentrations between the 2 treatment groups at baseline. At baseline, the infants in both groups were stunted and wasted: their weight-for-age, height-for-age, and weight-for-height $\mathrm{z}$ scores were all $<$ 0.(24). Criterion of a height-for-age z score $<-2$, was found in 110 (30.4\%) of the 331 subjects. Despite random allocation to treatment, there was an imbalance in the distribution of sexes in the treatment groups: boys were underrepresented in the intervention group [121 of 331 (39\%) compared with 185 of 331 (56\%) in the drops group]. However, hemoglobin values were not correlated with sex at baseline or at 8 - $16 \mathrm{mo}$. On the other hand, No treatment effect was found for weight-for-age or weight-for-height $\mathrm{z}$ scores in both groups from baseline to the final measurements (change: $0.16 \pm 0.65 ; \mathrm{P}<0.04$ ).

\section{Conclusion}

Much can be learned from this study about pragmatic approaches to reducing the prevalence of anemia: The results of this study indicate that, in a controlled setting, micronutrient Sprinkles result in a rate of successful treatment of anemia comparable with the current standard form-ferrous sulfate drops. Low dose Sprinkles (12.5 $\mathrm{mg}$ ) is as effective as higher doses of Sprinkles or iron drops in raising hemoglobin levels in anemic children. Low dose Sprinkles (12.5 mg) has fewer side effects and better compliance compared to iron drops. Improving the safety, adherence, sustainability and cost-effectiveness of iron supplementation is a continued goal for researches around the world. The option of using once daily drops or Sprinkles, therefore, may improve adherence to treatment and thus the success rate for the prevention and treatment of anemia. In the future, micro-encapsulated iron Sprinkles may be useful strategy for treatment and prevention of iron deficiency in infants because they are simple to use, lack side effects and are as efficacious as drops. Additional appropriately designed research is needed to determine the likely effectiveness of the Sprinkles in a non-study setting. We believe that Sprinkles will be widely accepted and preferable to iron drops as a means of iron supplementation if they are promoted in this population.

\section{Acknowledgements}

We gratefully acknowledge the dedicated and excellent fieldwork for their hard working. We are also thankful to all the infants and their parents for their participation in the study. At the same time, we thank the University of Al-Yemenia for funding the project particularly, Vic-Chancellor of University; Professor Abdul Wahed AlZindani for his continues moral support.

\section{References}

[1] Kapur, D., Agarwal, K.N., Sharma S., Kela, K. and Kaur, I. (2002) Iron Status of Children Aged 9 - 36 Months in an Urban Slum Integrated Child Development Services Project in Delhi. Indian Pediatric, 39, 136-144.

[2] Verma, M., Chhatwal, J. and Kaur, G. (1998) Prevalence of Anemia among Urban School Children of Punjab. Indian Pediatric, 35, 1181-1186.

[3] Zlotkin, S. (2002) Current Issues for the Prevention and Treatment of Iron Deficiency Anemia. Indian Pediatric, 39, 125-129.

[4] Zlotkin, S.H., Schauer, C., Christofides, A., Sharieff, W. and Hyder, S.M. (2005) Micronutrient Sprinkles to Control Childhood Anemia: A Simple Powdered Sachet May Be the Key to Addressing a Global Problem. PLoS Med, 2 , e1.

[5] Liyanage, C. and Zlotkin, S. (2002) Bioavailability of Iron from Micro-Encapsulated Iron Sprinkle Supplement. Food and Nutrition Bulletin, 23, 133-137. 
[6] Zlotkin, S., Arthur, P., Antwi, K.Y. and Yeung, G. (2001) Treatment of Anemia with Microencapsulated Ferrous Fumarate Plus Ascorbic Acid Supplied as Sprinkles to Complementary (Weaning) Foods. The American Journal of Clinical Nutrition, 74, 791-795.

[7] Zlotkin, S., Antwi, K.Y., Schauer, C. and Yeung, G. (2003) Use of Microencapsulated Iron (II) Fumarate Sprinkles to Prevent Recurrence of Anemia in Infants and Young Children at High Risk. Bulletin of the World Health Organization, 81, 108-115.

[8] Schauer, C. and Zlotkin, S. (2003) Home Fortification with Micronutrient Sprinkles-A New Approach for the Prevention and Treatment of Nutritional Anemia. Pediatric Child Health, 8, 87-90.

[9] World Health Organization (2000) Malnutrition. The Global Picture. World Health Organization, Geneva.

[10] Oski, F.A., Honig, A.S., Helu, B. and Howanitz, P. (1983) Effect of Iron Therapy on Behavior Performance in Nonanemic, Iron-Deficient Infants. Pediatrics, 71, 877-880.

[11] Nestel, P. and Alnwick, D. (1996) Iron/Multi-Micronutrient Supplements for Young Children. Summary and Conclusions of a Consultation Held at UNICEF, Copenhagen.

[12] Tondeur, M.C., Schauer, C., Christofides, A.L., Asante, K.P. and Newton, S. (2004) Determination of Iron Absorption from Intrinsically Labeled Microencapsulated Ferrous Fumarate (Sprinkles) in Infants with Varying Iron/Hematologic Status Using a Dual Stable Isotope Method. The American Journal of Clinical Nutrition, 80, 1436-1444.

[13] Zlotkin, S.H., Christofides, A., Schauer, C., Asante, K.P. and Owusu-Agyei, S. (2004) Home Fortification Using Sprinkles Containing 12.5 mg of Iron Successfully Treats Anemia in Ghanian Infants and Young Children. FASEB Journal, 18.

[14] Galloway, R. and McGuire, J. (1994) Determinants of Compliance with Iron Supplements: Supplies, Side Effects or Psychology. Social Science \& Medicine, 39, 381-390. http://dx.doi.org/10.1016/0277-9536(94)90135-X

[15] Davidsson, L., Kastenmayer, P., Szajewska, H., Harrell, R.F. and Barclay, D. (2000) Iron Bioavailability in Infants from an Infant Cereal Fortified with Ferric Pyrophosphate or Ferrous Fumarate. The American Journal of Clinical Nutrition, 71, 597-602.

[16] Heinrich, H.C., Gabbe, E.E., Whang, D.H., Bender-Gotze, C. and Schafer, K.H. (1975) Ferrous and Hemoglobin-59Fe Absorption from Supplemented Cow's Milk in Infants with Normal and Depleted Iron Stores. Zeitschrift für Kinderheilkunde, 120, 251-257. http://dx.doi.org/10.1007/BF00440264

[17] Cohen, A.R. and Seidl-Friedman, J. (1988) HemoCue System for Hemoglobin Measurement. Evaluation in Anemic and Nonanemic Children. American Journal of Clinical Pathology, 90, 302-305.

[18] Miles, L.M., Lipchitz, D.A., Bibber, C.P. and Cook, J.D. (1974) Measurement of Serum Ferritin by a 2-Site Immunoradiometric Assay. Anal Biochem, 61, 209-224. http://dx.doi.org/10.1016/0003-2697(74)90347-9

[19] Zlotkin, S.H., Arthur, P., Antiri, K.Y. and Yeung, G. (2001) Treatment of Anemia with Microencapsulated Ferrous Fumarate Plus Ascorbic and Supplied as "Sprinkles" to Complementary (Weaning) Foods. The American Journal of Clinical Nutrition, 74, 791-795.

[20] Rajurkar, N.S., Patil, S.F. and Zatakia, N.H. (2012) Assessment of Iron and Hemoglobin Status in Working Women of Various Age Groups. Journal of Chemical and Pharmaceutical Research, 4, 2300-2305.

[21] Gangadhar, T., Srikanth, P. and Suneetha, Y. (2010) Predictive Value of Iron Store Markers in Anemia of Chronic Kidney Disease. Journal of Chemical and Pharmaceutical Research, 2, 400-410.

[22] Yazar, H. and Kayhan, B.C. (2010) Adult Hemodialysis Patients with End-Stage Renal Disease and Erythropoietin Treatment of the Relation between Hypertension. Journal of Chemical and Pharmaceutical Research, 2, 588-593.

[23] Lozoff, B., Brittenham, G.M. and Wolf, A.W. (1987) Iron Deficiency Anemia and Iron Therapy Effect on Infants Developmental Test Performance. Pediatrics, 79, 981-993.

[24] AL-Jabri, F.M. and Mosa, E.Sh. (2012) Second Derivative Differential Electrolytic Potentiometers for Oxidation Reduction Reactions. Journal of Chemical and Pharmaceutical Research, 4, 2180-2187. 
Scientific Research Publishing (SCIRP) is one of the largest Open Access journal publishers. It is currently publishing more than 200 open access, online, peer-reviewed journals covering a wide range of academic disciplines. SCIRP serves the worldwide academic communities and contributes to the progress and application of science with its publication.

Other selected journals from SCIRP are listed as below. Submit your manuscript to us via either submit@scirp.org or Online Submission Portal.
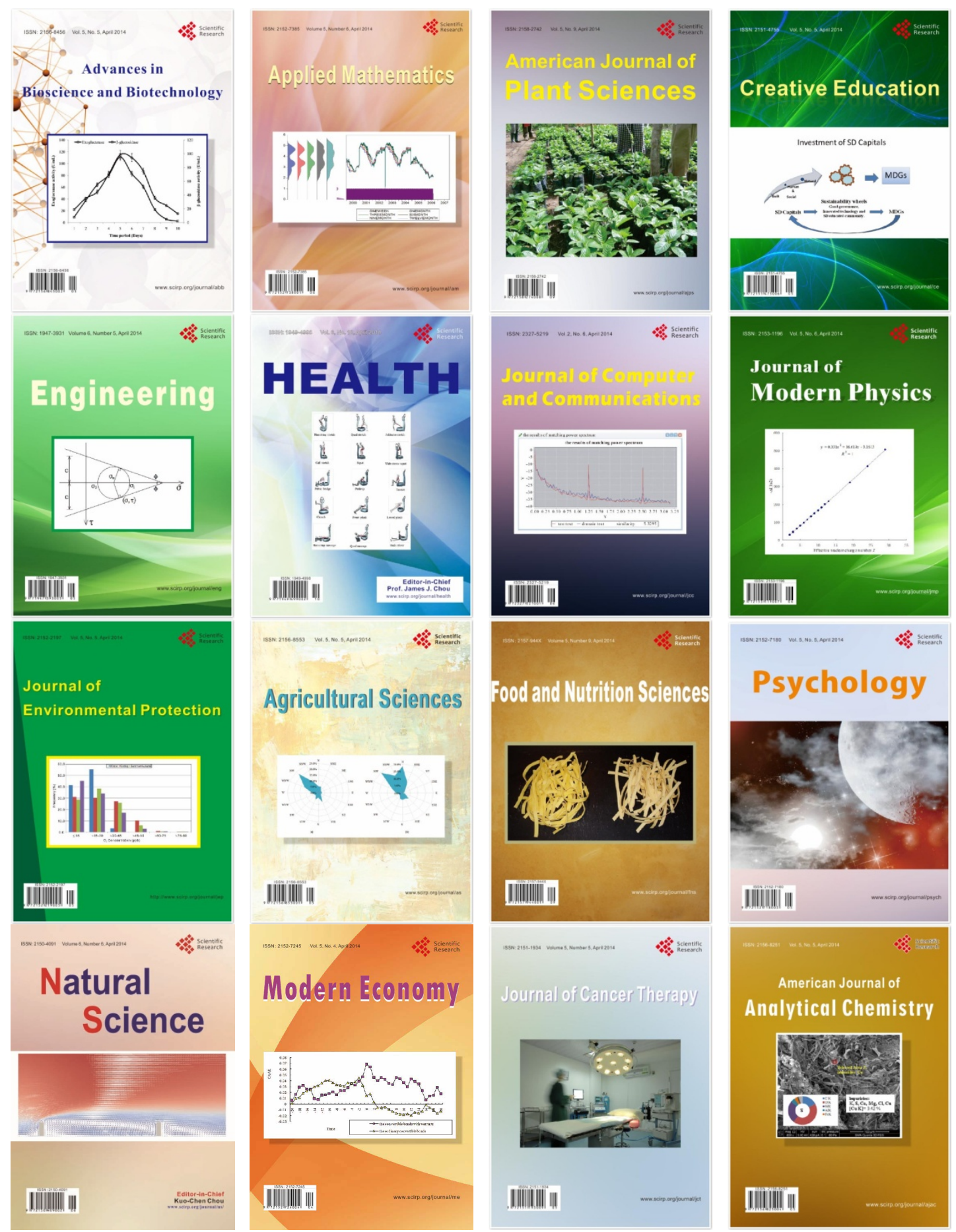